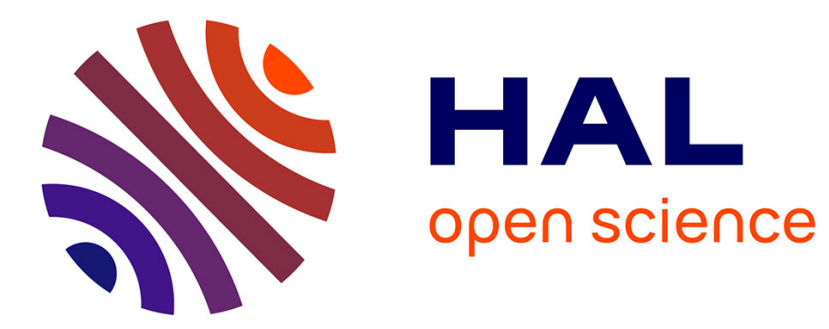

\title{
Study of data fusion algorithms applied to unattended ground sensor network
}

B. Pannetier, J. Moras, J. Dezert, G. Sella

\section{To cite this version:}

B. Pannetier, J. Moras, J. Dezert, G. Sella. Study of data fusion algorithms applied to unattended ground sensor network. SPIE Defense \& Security, May 2014, BALTIMORE, United States. hal01070377

\section{HAL Id: hal-01070377 \\ https://hal-onera.archives-ouvertes.fr/hal-01070377}

Submitted on 1 Oct 2014

HAL is a multi-disciplinary open access archive for the deposit and dissemination of scientific research documents, whether they are published or not. The documents may come from teaching and research institutions in France or abroad, or from public or private research centers.
L'archive ouverte pluridisciplinaire HAL, est destinée au dépôt et à la diffusion de documents scientifiques de niveau recherche, publiés ou non, émanant des établissements d'enseignement et de recherche français ou étrangers, des laboratoires publics ou privés. 


\title{
Study of data fusion algorithms applied to unattended ground sensor network
}

\author{
Pannetier B. ${ }^{a}$, Moras J. ${ }^{a}$, Dezert J. ${ }^{a}$ and Sella G. ${ }^{b}$ \\ ${ }^{a}$ ONERA, the French Aerospace Lab, Information processing and modelling department, \\ F-91761 Palaiseau, France; \\ ${ }^{b}$ SAGEM, Defence and optic division, CED CV, 100 avenue de Paris, 91344 Massy cedex, \\ France;
}

\begin{abstract}
In this paper, data obtained from wireless unattended ground sensor network are used for tracking multiple ground targets (vehicles, pedestrians and animals) moving on and off the road network. The goal of the study is to evaluate several data fusion algorithms to select the best approach to establish the tactical situational awareness. The ground sensor network is composed of heterogeneous sensors (optronic, radar, seismic, acoustic, magnetic sensors) and data fusion nodes. The fusion nodes are small hardware platforms placed on the surveillance area that communicate together. In order to satisfy operational needs and the limited communication bandwidth between the nodes, we study several data fusion algorithms to track and classify targets in real time. A multiple targets tracking (MTT) algorithm is integrated in each data fusion node taking into account embedded constraint. The choice of the MTT algorithm is motivated by the limit of the chosen technology. In the fusion nodes, the distributed MTT algorithm exploits the road network information in order to constrain the multiple dynamic models. Then, a variable structure interacting multiple model (VS-IMM) is adapted with the road network topology. This algorithm is well-known in centralized architecture, but it implies a modification of other data fusion algorithms to preserve the performances of the tracking under constraints. Based on such VS-IMM MTT algorithm, we adapt classical data fusion techniques to make it working in three architectures: centralized, distributed and hierarchical. The sensors measurements are considered asynchronous, but the fusion steps are synchronized on all sensors. Performances of data fusion algorithms are evaluated using simulated data and also validated on real data. The scenarios under analysis contain multiple targets with close and crossing trajectories involving data association uncertainties.
\end{abstract}

Keywords: Multiple target tracking, wireless sensor network, data fusion architecture

\section{INTRODUCTION}

The goal of the work presented in this paper is to study and develop in the next years a new generation od operational wireless sensor networks (WSN) which consists of large number of smart heterogeneous sensors with onboard sensing, processing and wireless communication capabilities. The future operational WSN must satisfy severe exigencies in term of survivability (few weeks), low communications (to be undetectable by communication interception system), and real-time tactical situation assessment for large surveillance areas. The use of WSN network must also be easy and remotely controllable and have a low cost. The system must be easy to deploy, implemented by a limited number of operators with a minimum training through a simple human machine interface (HMI) for its exploitation and for decision-making support. Finally, the system must be modular, flexible and dynamically configurable (depending on the environment, the threat and mission). The main system characteristics of such system are:

Further author information: (Send correspondence to Benjamin Pannetier)

B.P.: E-mail: benjamin.pannetier@onera.fr, Telephone: +33 (0)1 80386578

J.M.: E-mail: julien.moras@onera.fr, Telephone: +33 (0)1 80386543

J.D. : E-mail: jean.dezert@onera.fr, Telephone: +33 (0)1 80386564

G.S. : E-mail: genevieve.sella@sagem.com, Telephone: +33 (0)1 58119728 
- efficiency: the system must provide highest performances,

- modularity and operational flexibility,

- reliability: failures must be detected, isolated and fixed by sensor substitution (when possible),

- real-time use: information must be received and precessed in real-time for the operational need,

- survivability: besides camouflage and discretion of the means deployed, optimizing the energy and the network resistance to aggression is a problem for the operational resilience,

- affordability.

Components (both sensors and communications devices) must have low energy consumptions, to be able to work in a remote mode, in an outdoor environment and to fulfill discretion constraints required to work in unattended operating modes. The system must be easy to deploy and be able to adapt to various natures of terrain and topographies.

Our demonstrator is intended to allow studies on automatic data processing with an objective to correlate detection and generate only one alert on each target, being tracked as time goes on. It will allow us to evaluate several schemes for the data collection and fusion process, and to demonstrate the necessity of taking into account high-level information (typically geographic information, as traffic lanes, intersections, areas without terrain obscuration,...) for deployment and exploitation of the system.

Several processing levels are considered in this work:

- local processing of raw data at the sensor level: it can provide a detection alert on the presence of a target, and eventually some attributes about the target (as target location and type),

- additional processing on raw data (as basic image processing on sensor nodes),

- data fusion on a sensor node from a set of information collected from other sensors (target kinematics (e.g. tracks), classifications, their number, etc).

In this paper, we study the problem of tracking multiple moving objects observed through a WSN with limited sensing abilities. Our purpose is to track several targets in maintaining high track continuity performance to provide a reliable situation assessment. For this goal, we use heterogeneous sensors to compensate the low amount of data available (due to the weak sensor area coverage) by a better information quality on the data (both in precision of location and in classification information). The proposed data sensor processing presented in this work allows to meet the operational needs.

Several papers have been published on operational sensor processing applied to WSN. For example, Ekman and Pålson described in ${ }^{1}$ a modified particle filter $(\mathrm{PF})^{2}$ to track a single vehicle through the WSN. Similar approach can be found in. ${ }^{3}$ Despite of the well known estimation performances due to the generation of the particles on the road network, we haven't selected a PF algorithm because we need to track several targets in the sensor network with severe processing constraints due to hardware solution used in our demonstrator to preserve the power of a fusion node. In fact, because PF approach uses more CPU than Kalman filter (KF), extended Kalman filter (EKF) or unscented Kalman filter (UKF), we cannot use it in our specific context if one wants to make the surveillance system operational during a long period of time. Parmar and Zaveri in ${ }^{4}$ have done similar studies and achieve the same conclusions. They focus their study of the data association for MTT in WSN and the need to limit the power to maintain the WSN in activity during a long time. However, if the hardware performances are improved with the advances of technology to meet the power constraint, the use of $\mathrm{PF}$ will become possible for this application. In fact, $\mathrm{Oh}$ and al. described in $\overline{5}$ a complete $\mathrm{PF}$ algorithm (called MCMCDA algorithm) applied for tracking multiple targets in a WSN with communication constraints. To improve the MTT algorithm performance, we introduce in this work the geographic informations in the tracking process as proposed by Ulmke and Koch in. ${ }^{6}$ Since we want to track both ground vehicles (that can move on and off the road), aerial vehicles that are not constrained on the road, and pedestrians as well, we use on-road 


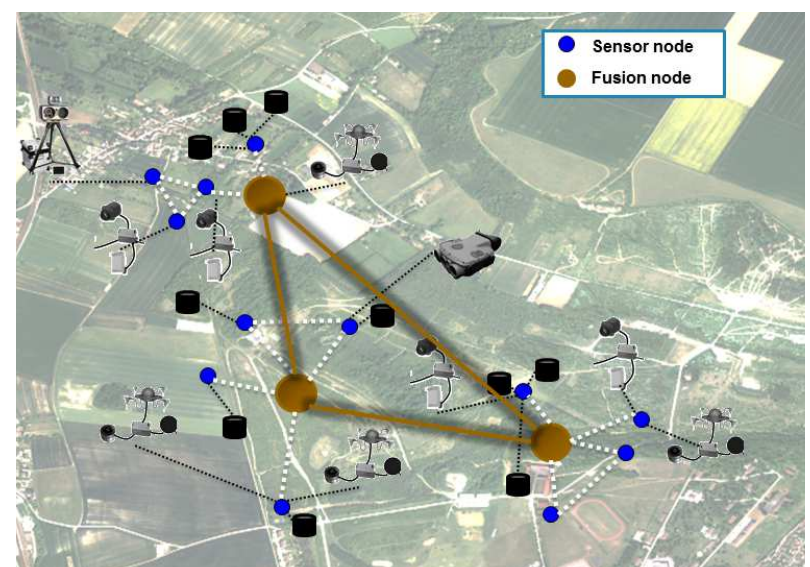

Figure 1: Sensor network architecture.

and off-road tracking algorithms. For doing this, we have adapted the MTT ground target tracking algorithm described in ${ }^{7}$ for our WSN tracking demonstrator.

The paper is organized as follows : in section 2 the WSN is briefly presented. Section 3 describes the multiple motion model algorithm constrained to geographic information. Section 4 presents the architectures to track multiple target. Results from the study are given in section 5. Finally, concluding remarks are presented in section 6 .

\section{WIRELESS SENSOR NETWORK DESCRIPTION}

\subsection{Network description}

The good quality of communication between the sensor nodes has a strong impact on the ability of WSN to fulfill its task of surveillance. It is also very important that the WSN can communicate with the Command and Control (C2) station. The solution proposed in this paper is based on on-the-shelf existing components. Its multi-cluster architecture is represented in figure 1.

This architecture is structured in two levels:

- a set of clusters: sensor and fusion nodes connected through a low energy, low Rate 802.15.4 wireless network, managed by a gateway;

- a backbone with higher rate gathering data from clusters which guarantees the expected connectivity and allows two-ways communications.

The main information transmitted on the network are the following: data from sensor to sensor-nodes and to $\mathrm{C} 2$, state of the components to sensor node and to $\mathrm{C} 2$, command to sensors from $\mathrm{C} 2$ or sensor node to components, exchange between sensor nodes to allow horizontal data fusion. Two categories of sensors: low consumption sensors that can be kept in operation to provide a continuous surveillance, and sensors having higher consumption that can be activated in case of presence of a target to acquire more detailed information on it.

The sensor node receives data from other sensors, processes them and transmits the local result to the fusion node. A set of complementary sensors is selected in order to collect multi-spectral information from the threats. These information will be used in order to

- detect the presence of a target, or an event, 
- provide a spatial location of the event: sensors provide at current time $t_{k}$ a measurement $\mathbf{z}_{k}$ (bearing $\theta_{k}$, elevation $\phi_{k}$, distance $\rho_{k}$ and radial velocity $\dot{\rho}_{k}$ ) in the sensor reference frame. Most sensors are able to give only partial location: bearing and distance only for radars, bearing and elevation for electro-optical sensors,

- classify the nature of event among the given set of classes $C$. The output of the classification process is a vector $\mathbf{c}_{k}$, where each component is the likelihood of each target class. Typically, we consider the following set of classes

$$
\begin{array}{r}
C=\{\text { light-vehicle, } \text { heavy-vehicle, } \text { tracked-vehicle, } \\
\text { human, people, aerial targets }\}
\end{array}
$$

The set of classes $C$ imposed by the project requirement is not conventional because the class heavy-vehicle and tracked-vehicle are not exclusive, as well as the class class human which is included in the people (human group) class. The human class is sub-class (a singleton) of the people class. That is why we have proposed at the sensor level for light-vehicle and heavy-vehicle classes to discriminate with sub-classes tracked-light-vehicle, wheeled-light-vehicle and tracked-heavy-vehicle, wheeled-heavy-vehicle respectively.

Different video algorithms have been studied at ONERA and we have integrated one of them in the sensor node to detect, localize and classify automatically the targets with the previous considerations. The result of the processing (event, detection and classification information) is emitted to the fusion node. The same kind of process applies with acoustic sensors.

\subsection{Sensor model}

The generic observation model for the sensor no. $j$ is given by:

$$
\mathbf{z}_{k}^{j}=h^{j}\left(\mathbf{x}_{k}\right)+\mathbf{b}_{k}^{j}
$$

where $h^{j}(\cdot)$ is the observation function, $\mathbf{x}_{k}$ is the state of a target (detailed in the next section), and $\mathbf{b}_{k}^{j}$ is a zero-mean white Gaussian noise vector with a known covariance matrix $\mathbf{R}_{k}^{j}$. The observation function and the associated noise depends on the type of sensor. We distinguish three observations functions : $h^{\text {radar }}, h^{a c o u}$, $h^{\text {optro }}, h^{\text {mag }}$ associated respectively to the radar, acoustic, optic and magnetic sensors.

$$
\begin{aligned}
& h^{\text {radar }}\left(\mathbf{x}_{k}\right)=\left[\begin{array}{lll}
\rho_{k} & \theta_{k} & \dot{\rho}_{k}
\end{array}\right]^{\prime} h^{\text {acou }}\left(\mathbf{x}_{k}\right)=\left[\theta_{k}\right] \\
& h^{o p t}\left(\mathbf{x}_{k}\right)=\left[\begin{array}{ll}
\theta_{k} & \phi_{k}
\end{array}\right] h^{m a g}\left(\mathbf{x}_{k}\right)=\left[\begin{array}{ll}
x_{k} & y_{k}
\end{array}\right]
\end{aligned}
$$

For the magnetic sensor, we use its own location in the topographic coordinated frame (TCF), described in the next part, in order to model a measurement because of its short range detection (see Table 1).

The different types of sensor that can be connected to a sensor node are listed in Table 1 The Volume indicates the area coverage where the target can be found. This event is emitted as well as measurement to the fusion node in order to correlate this information with another volume, or a sensor detection to get a localized detection in the TCF.

\subsection{Localization step}

The localization module is used to localize all sensors and data in the TCF. For doing this, we need a calibration of each sensor. Several calibration techniques will be tested during the experimental trials, based on specific devices (GPS, DGPS) allowing measurement of position and orientation of individual components, on cooperative localization using range or direction measurements between two sensors nodes, and on specific methods for calibration of electro-optics sensors.

For the sensors providing only volume information, or bearing detection, the localization module exploits all available information on sensors and elementary detection to provide a composite report $\mathbf{z}^{\text {comp }}(k)$ in the TCF that will feed the data fusion process. The sensors provide detections and information on location of the target 


\begin{tabular}{|c|c|c|c|}
\hline Sensor type & number & $\begin{array}{c}\text { sensor node } \\
\text { output }\end{array}$ & $\begin{array}{c}\text { detection } \\
\text { characteristic }\end{array}$ \\
\hline acoustic antenna & 3 & $\begin{array}{l}\theta_{k} \\
\mathbf{c}_{k}\end{array}$ & $\begin{array}{l}\text { spherical } \\
<200 \mathrm{~m}\end{array}$ \\
\hline acoustic beacon & 4 & $\begin{array}{l}\text { Volume } \\
\mathbf{c}_{k}\end{array}$ & $\begin{array}{l}\text { spherical } \\
<200 \mathrm{~m} \\
\end{array}$ \\
\hline magnetic & 10 & Volume $_{\mathbf{c}_{k}}$ & $\begin{array}{l}\text { spherical } \\
<2 \mathrm{~m}\end{array}$ \\
\hline radar & 1 & $\rho_{k}, \theta_{k}, \dot{\rho}_{k}$ & $\begin{array}{l}\text { Sectoral }=90^{\circ} \\
\quad<1000 \mathrm{~m}\end{array}$ \\
\hline PIR & 8 & $\theta_{k}$ & $\begin{array}{c}\text { mono,multi beam } \\
<200 \mathrm{~m}\end{array}$ \\
\hline $\begin{array}{c}\text { micro-camera } \\
\text { UIR }\end{array}$ & 4 & $\begin{array}{c}\theta_{k}, \phi_{k} \\
\mathbf{c}_{k}\end{array}$ & $\begin{array}{c}\text { Sectoral }=30^{\circ}, 40^{\circ} \\
<100,200 \mathrm{~m}\end{array}$ \\
\hline $\begin{array}{c}\text { FIR short } \\
\text { UIR+visible }\end{array}$ & 1 & $\begin{array}{c}\theta_{k}, \phi_{k} \\
\mathbf{c}_{k} \\
\end{array}$ & $\begin{array}{l}\text { Sectoral }=10^{\circ} \\
<100,200 \mathrm{~m}\end{array}$ \\
\hline $\begin{array}{c}\text { JIM LR } \\
\text { IR+visible }\end{array}$ & 1 & $\begin{array}{c}\theta_{k}, \phi_{k} \\
\mathbf{c}_{k}\end{array}$ & $\begin{array}{l}\text { Sectoral }=10^{\circ} \\
<100,200 \mathrm{~m}\end{array}$ \\
\hline $\begin{array}{l}\text { Cham } \\
\text { visible }\end{array}$ & 4 & $\begin{array}{c}\theta_{k}, \phi_{k} \\
\mathbf{c}_{k}\end{array}$ & $\begin{array}{c}\text { Sectoral }=5^{\circ}, \text { to } 50^{\circ} \\
<100,200 \mathrm{~m}\end{array}$ \\
\hline
\end{tabular}

Table 1: Types of sensors used in the demonstrator.

in their own reference frame. To work in common TCF for situation assessment we always need a calibration step.

For notation convenience, the measurements sequence at the fusion node $w(\forall w \in\{1, \ldots, W\})$, defined by $Z^{k, l}=\left\{Z^{k-1, n}, \mathbf{z}_{k}^{j_{w}}\right\}$ represents a possible set of measurements generated by the target up to time $k$. $Z^{k, l}$ consists of a subsequence $Z^{k-1, n}$ of measurements up to time $k-1$ and a validated measurement $\mathbf{z}_{k}^{j_{w}}$ available at time $k$ from sensor $j_{w}$ associated with the track $\mathcal{T}_{k, l}^{w}$. The sensor $j_{w}$ is connected to a node $w$. At the current time $k$, the track $\mathcal{T}_{k, l}^{w}$ is represented by a sequence of the state estimates.

\section{TARGET TRACKING WITH GEOGRAPHIC CONSTRAINT}

\subsection{Geographic Information System}

The geographic information system (GIS) used in this work contains the following information: the segmented road network, the hydrographic network, the vegetation area, the buildings area and DTED (Digital Terrain Elevation Data). Only the network and elevation information (the DTED + buildings height) are used in the first part of our study presented in this paper.

The road network is connected, and each road segment is indexed by the road section it belongs to. A road section is defined by a finite set of connected road segments delimited by a road end or a junction. For the topographic information, we use the database called: BD TOPO* This GIS has a metric precision on the road-segments location.

At the beginning of a surveillance battlefield operation, a TCF and its origin $O$ are chosen in the manner that the axes $X, Y$ and $Z$ are respectively oriented in the East, North and Up local direction. The target tracking process is carried out in the TCF. In addition, starting from the elevation terrain and the sensor location at the current time, it is possible to compute the perceivability $P_{e}$ at any referenced point for a sensor $j_{w}$. In the sequel, $P_{e}^{j_{w}}(x, y, k)$ will denote the probability for the sensor $j_{w}$ to detect at time $k$ a target at the location $(x, y)$.

\footnotetext{
* See www.professionnels.ign.fr/bdtopo for a description of this GIS.
} 


\subsection{Context constraint tracking}

The target state at the current time $t_{k}$ is defined in the local horizontal plane of the TCF by the vector:

$$
\mathbf{x}_{k}=\left[\begin{array}{llll}
x_{k} & \dot{x}_{k} & y_{k} & \dot{y}_{k}
\end{array}\right]^{T}
$$

where $\left(x_{k}, y_{k}\right)$ and $\left(\dot{x}_{k}, \dot{y}_{k}\right)$ define respectively the target location and velocity in the local horizontal plane.

The dynamics of the target evolving on the road are modeled by a first-order plant equation. The target state on the road segment $s$ is defined by $\mathbf{x}_{k}^{s}$ where the target position $\left(x_{k}^{s}, y_{k}^{s}\right)$ belongs to the road segment $s$ and the corresponding heading $\left(\dot{x}_{k}^{s}, \dot{y}_{k}^{s}\right)$ in its direction.

The event that the target is on road segment $s$ is noted $e_{k}^{s}=\left\{\mathbf{x}_{k} \in s\right\}$. Given this event $e_{k}^{s}$ and according to a motion model $\mathcal{M}_{i}$, the estimation of the target state can be improved by considering the road segment $s$. For a constant velocity motion model, it follows:

$$
\mathbf{x}_{k}^{s}=\mathbf{F}^{s, i}\left(\Delta_{k}\right) \cdot \mathbf{x}_{k-1}^{s}+\boldsymbol{\Gamma}\left(\Delta_{k}\right) \cdot \mathbf{v}_{k}^{s, i}
$$

where $\Delta_{k}$ is the sampling time, $\mathbf{F}^{s, i}$ is the state transition matrix associated to the road segment $s$ and adapted to a motion model $\mathcal{M}_{i} ; \mathbf{v}_{k}^{s, i}$ is a white zero-mean Gaussian random vector with covariance matrix $\mathbf{Q}_{k}^{s, i}$ chosen in such a way that the standard deviation $\sigma_{d}$ along the road segment is higher than the standard deviation $\sigma_{n}$ in the orthogonal direction. It is defined by:

$$
\mathbf{Q}_{k}^{s, i}=\mathbf{R}_{\theta_{s}} \cdot\left(\begin{array}{cc}
\sigma_{d}^{2} & 0 \\
0 & \sigma_{n}^{2}
\end{array}\right) \cdot \mathbf{R}_{\theta_{s}}^{T}
$$

where $\mathbf{R}_{\theta_{s}}$ is the rotation matrix associated with the direction $\theta_{s}$ defined in the plane $(O, X, Y)$ of the road segment $s$. The matrix $\boldsymbol{\Gamma}\left(\Delta_{k}\right)$ is defined in.

To improve the modeling for targets moving on a road network, we have proposed in ${ }^{9}$ to adapt the level of the dynamic model's noise based on the length of the road segment $s$. The idea is to increase the standard deviation $\sigma_{n}$ defined in $(\sqrt{6})$ ) to take into account the error on the road segment location. After the state estimation obtained by a Kalman filter, the estimated state is then projected according to the road constraint $e_{k}^{s}$. This step is detailed in $\underline{10}$

\subsection{Fusion node IMM under road segment constraint}

Here we recall briefly the principle of the interacting multiple model (IMM) taking into account the road network constraints. The IMM is a well-known efficient maneuvering target tracking algorithm ${ }^{11}$ which combines estimated states based on multiple models to get a better global state estimate. The IMM is near optimal and has a reasonable complexity which makes it very appealing in tracking applications. In section 3.2, a constrained motion model $i$ to segment $s$, noted $\mathcal{M}_{k}^{s, i}$, was defined. There is a distinction between the definition of a motion model $\mathcal{M}_{k}^{s, i}$ (i.e. motion model type, noise,...) and the event $M_{k}^{s, i}$ that the target is moving on the road according the motion model $i$ at time $k$. Here we extend the segment constraint to the different dynamic models (among a set of $r+1$ motion models) that a target can follow. The model indexed by $r=0$ is the stop model. The transition between the models is modelled as a Markovian process. In general when the target moves from one segment to the next, the set of dynamic models changes. In a conventional IMM estimator $\frac{11}{11}$ the likelihood function of a model $i$ is given, for a track $\mathcal{T}_{k, l}^{w}$, associated with the $j_{w}$-th measurement, $j_{w} \in\left\{0,1, \ldots, m_{k}\right\}$ by:

$$
\Lambda_{k}^{i}=p\left\{\mathbf{z}_{k}^{j_{w}} \mid M_{k}^{s, i}, Z^{k-1, n}\right\}, \quad i=0,1, \ldots, r
$$

where $Z^{k-1, n}$ is the subsequence of measurements associated with the track $\mathcal{T}_{k, l}^{w}$.

Using the IMM estimator with a stop-motion model, we get the likelihood function of the moving target mode for indexes $i \in\{0,1, \ldots, r\}$ and for $j_{w} \in\left\{0,1, \ldots, m_{k}\right\}$ by:

$$
\Lambda_{k}^{i}=P_{D} \cdot p\left\{\mathbf{z}_{k}^{j_{w}} \mid M_{k}^{s, i}, Z^{k-1, n}\right\} \cdot\left(1-\delta_{j_{w}, 0}\right)+\left(1-P_{D}\right) \cdot \delta_{j_{w}, 0}
$$




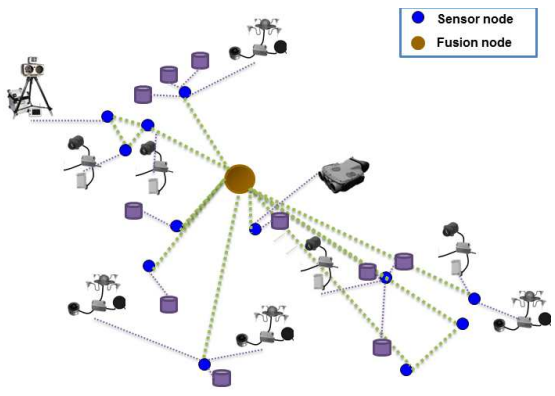

(a) Centralised architecture.

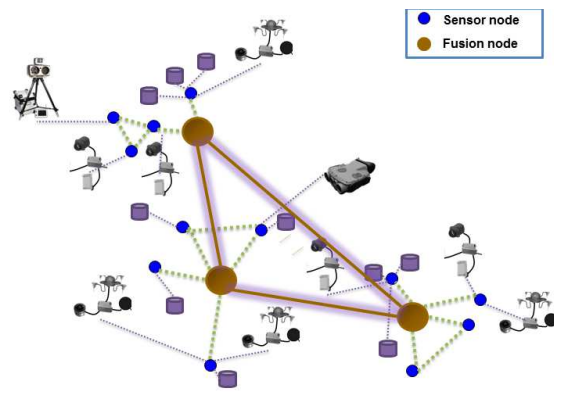

(b) Ditributed architecture.

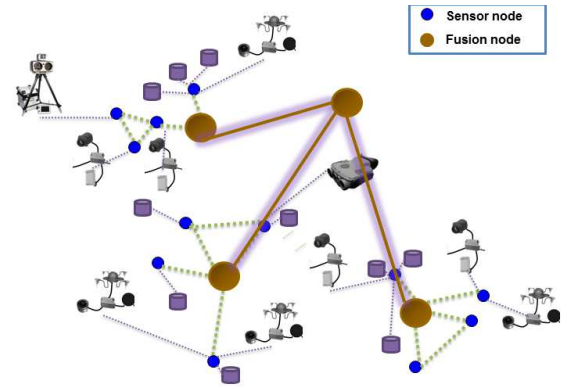

(c) Hierarchical architecture.

Figure 2: Tested data fusion architecture.

The likelihood of the stopped target mode (i.e. $r=0)$ is:

$$
\Lambda_{k}^{0}=p\left\{\mathbf{z}_{k}^{j_{w}} \mid M_{k}^{s, 0}, Z^{k-1, n}\right\}=\delta_{j, 0}
$$

where $\delta_{j_{w}, 0}$ is the Kronecker function defined by $\delta_{j_{w}, 0}=1$ if $j=0$ and $\delta_{j_{w}, 0}=0$ otherwise.

The combined (global) likelihood function $\Lambda_{k}$ of a track including a stop-motion model is then given by:

$$
\Lambda_{k}=\sum_{i=0}^{r} \Lambda_{k}^{i} \cdot \mu_{k \mid k-1}^{i}
$$

where $\mu_{k \mid k-1}^{i}$ is the predicted model probabilities. The steps of the IMM under road segment $s$ constraint are the same as for the classical IMM and it has been described in .9

Here, one has used the IMM algorithm constrained to only one road segment $s$. However, a road section is composed with several road segments. When the target is making a transition from one segment to another, the problem is to choose the segments with the corresponding motion models that can better fit the target dynamics. The choice of a segment implies the construction of the directional process noise. That is why the IMM motions model set varies with the road network configuration and a variable-structure IMM (VS IMM) offers a better solution for ground target tracking on road networks. Such algorithm has been denoted VS IMMC (C standing for Constrained) and presented in details in! 12

\section{DATA FUSION ARCHITECTURES}

In order to limit communication between fusion nodes and maintain the multiple target tracking performance, we study in this part several fusion node architectures. The first one is the the centralized architecture (figure 2a in which all the data acquired by the sensors are transmitted by the sensor nodes to only one fusion node. This architecture, known to provide optimal results, suffers of the large amount of data passing through the network and it presents a serious weakness against threats and attacks The second architecture is the distributed architecture (figure 2b) which presents the advantage to require between fusion nodes only the minimal amount of useful information for updating the local tracks. Each fusion node must have the same tactical situation. The third architecture is the hierarchical architecture (figure 2c) which has the advantage of offering a low communication exigency between fusion nodes, but in this architecture only the master fusion nodes has a complete tactical situation representation.

\subsection{Multiple target tracking in centralized architecture}

The MTT algorithm that we use in a fusion node for the centralized architecture is the SB-MHT associated the the previous VS IMM. More details can be found in Blackman-Popoli's book ${ }^{8}$ (chapter 16). We briefly describe here the main steps 
1. The first functional block of the SB-MHT is the track confirmation and the track maintenance. When the new set $Z^{k}$ of measurements is received, a standard gating procedure ${ }^{8}$ is applied in order to determine the valid measurement reports to track pairings. The existing tracks are updated with VS IMMC and extrapolated confirmed tracks are formed. When the track is not updated with reports, the stop-model is activated.

2. In order to palliate the association problem, we need a probabilistic expression for the evaluation of the track formation hypotheses that includes all aspects of the data association problem. It is convenient to use the LLR or track score of a track $\mathcal{T}_{k, l}^{w}$ which can be expressed at current time $k$ in the following recursive form: $[8$

$$
L_{k, l}=L_{k-1, n}+\Delta L_{k, l}
$$

with

$$
\Delta L_{k, l}=\log \left(\frac{\Lambda_{k}}{\lambda_{f a}}\right)
$$

and

$$
L(0)=\log \left(\frac{\lambda_{f a}}{\lambda_{f a}+\lambda_{n t}}\right)
$$

where $\lambda_{f a}$ and $\lambda_{n t}$ are respectively the false alarm rate and the new target rate per unit of surveillance volume. $\Lambda_{k}$ is the global likelihood function described in 100 . After the track score calculation of the track $\mathcal{T}_{k, l}^{w}$, the SPRT is used to set up the track status either as deleted, tentative or confirmed track. The tracks that fail the SPRT are deleted and the surviving tracks are kept for the next stage.

3. The process of clustering is to put altogether the tracks that are linked by a common measurement. The clustering technique is used to limit the number of hypotheses to generate, and therefore to reduce the complexity of tracking system. The result of the clustering process is a list of tracks that are interacting. The next step is to form hypotheses of compatible tracks.

4. For each cluster, multiple coherent hypotheses are formed to represent the different compatible tracks scenarios. Each hypothesis is evaluated according to the track score function associated to the different tracks. Then, a technique is required to find the set of hypotheses set that represents the most likely tracks collection. The unlikely hypotheses and associated tracks are deleted by a pruning process and only the $N_{\text {Hypo }}$ best hypotheses are conserved.

5. For each track, the a posteriori probability is computed and a classical $N$-Scan pruning approach ${ }^{8}$ is used to select the confirmed and delete the most unlikely tracks. With this approach the most likely tracks are selected to reduce the number of tracks. However, the $\mathrm{N}$-Scan technique combined with the constraint implies that other tracks hypotheses (i.e. constrained on other road segments) are arbitrary deleted. To avoid this problem, we modify the $N$-Scan pruning approach in order to select the $N_{k}$ best tracks on each $N_{k}$ road sections.

6. Wald's SPRT ${ }^{8}$ is used to delete the unlikely hypotheses among the $N_{k}$ hypotheses. The tracks are then updated and projected on the road network. In order to reduce the number of tracks to keep in the memory of the computer, a merging technique (selection of the most probable tracks which have common measurements) is also implemented.

\subsection{Track fusion in distributed architecture}

In this part we present briefly the well-known distributed interacting multiple model (DIMM) introduced by Ding and Hong! 13 The choice of this algorithm is motivated by the VS IMMC used in the part 3 . The DIMM is structured in three levels plus one for the track association for the fusion. 
Each fusion node $w,(\forall w \in\{1, \ldots, W\})$, produces local tracks $\mathcal{T}_{k, l}^{w}$ with their own sensors according the VS IMMC SB-MHT. This is a "local" centralized fusion node.

1. Fusion node equivalent target model. Before the emission of the updated tracks $\mathcal{T}_{k, l}^{w}$ trough the network, the fusion node calculate the equivalent target model to modelize the information used to estimate the state $\hat{\mathbf{x}}_{k \mid k}^{l, w}$ and its associated covariance $\mathbf{P}_{k \mid k}^{l, w}$. As presented in Ding's paper ${ }^{13}$ a fusion node equivalent target model for each fusion node $w(\forall w \in\{1, \ldots, W\})$ is given by:

$$
\mathbf{x}_{w, k}=\mathbf{F}_{w}\left(\Delta_{k}\right) \mathbf{x}_{w, k-1}+\mathbf{b}_{w}\left(\Delta_{k}\right)
$$

where $\mathbf{F}_{w}$ and $\mathbf{b}_{w}$ are respectively the equivalent transition matrix from the node $w$ and the equivalent white Gaussian noise process defined by the herein formulas:

$$
\mathbf{F}_{w}\left(\Delta_{k}\right)=\sum_{i=0}^{r} \mu_{k}^{i} \mathbf{F}^{s, i}\left(\Delta_{k}\right)
$$

where $\mu_{k}^{i}$ is the constraint motion model probability of the model $\mathcal{M}_{k}^{s, i}$ and $\mathbf{F}^{s, i}$ is the transition matrix of the model described in (5). Te noise process is given by:

$$
\mathbf{b}_{w}\left(\Delta_{k}\right)=\sum_{i=0}^{r} \mu_{k}^{i} \Gamma\left(\Delta_{k}\right) \mathbf{v}_{s, i}\left(\Delta_{k}\right)
$$

and by exploiting the noise independence between motion model, we obtain the noise covariance of the equivalent model:

$$
\mathbf{Q}_{w}\left(\Delta_{k}\right)=\sum_{i=0}^{r} \mu_{k}^{i 2} \mathbf{Q}_{k}^{s, i}
$$

This fusion node equivalent model is used to calculate the fusion node probability $\nu_{k}^{w}$ in each fusion node $w$.

2. Fusion node probability. In order to obtain the global equivalent model probability for each fusion node $w$, the fusion node probability $\nu_{w}$ must be calculated. The "Theoretical" $\left\{\left[\mathbf{z}_{k}^{j_{1}}, \ldots, \mathbf{z}_{k}^{j_{W}}\right], Z^{k-1}\right\}$ received at the fusion node is classically used to compute the model probability of the $w$-th fusion node $(\forall w \in\{1, \ldots, W\})$. The measurement $\mathbf{z}_{k}^{j_{w}}$ is the $j_{w}$-th measurement associated received at the fusion node $w$. The probability $\nu_{k}^{w}$ is given by:

$$
\nu_{k}^{w}=P\left\{N_{k}^{w} \mid Z^{k}\right\} \approx \frac{1}{c} \cdot p\left(\mathbf{z}_{k}^{j_{w}} \mid N_{k}^{w}, Z^{k-1}\right) P\left\{N_{k}^{w} \mid Z^{k-1}\right\}
$$

where $N_{k}^{w}$ is the event on the track estimation with the subsequence of measurement and $c$ is a normalizing constant. The likelihood function in eq. 18 can be expressed as:

$$
p\left(\mathbf{z}_{k}^{j_{w}} \mid N_{k}^{w}, Z^{k-1}\right)=\frac{1}{\left|2 \pi \mathbf{S}_{k}^{j_{w}}\right|^{\frac{1}{2}}} \exp \left(\mathbf{z}_{k}^{j_{w}}-\mathbf{H}_{k}^{j_{w}} \overline{\mathbf{x}}_{k \mid k-1}^{w}\right)^{\prime} \mathbf{S}_{k}^{j_{w}-1}\left(\mathbf{z}_{k}^{j_{w}}-\mathbf{H}_{k}^{w} \overline{\mathbf{x}}_{k \mid k-1}^{w}\right)
$$

where

$$
\mathbf{S}_{k}^{j_{w}}=\mathbf{H}_{k}^{j_{w}} \overline{\mathbf{P}}_{k \mid k-1}^{w} \mathbf{H}_{k}^{j_{w}{ }^{\prime}}+\mathbf{R}_{k}^{j_{w}}
$$

The prior fusion node probability in $(18)$ is given by:

$$
P\left\{N_{k}^{w} \mid Z^{k-1}\right\}=\sum_{w=1}^{W} \Pi_{j u m p}^{w i} \nu_{k-1}^{i}
$$

where $\Pi_{j u m p}^{w j}$ is the fusion node transition probability from fusion node $j$ to fusion node $w$. The predicted state $\overline{\mathbf{x}}_{k \mid k-1}^{w}$ and its associated covariance $\overline{\mathbf{P}}_{k \mid k-1}^{w}$ are obtained according the fusion node equivalent model (14).

\footnotetext{
${ }^{\dagger}$ In fact, the fusion nodes do not share measurements but tracks
} 
3. The fusion node model provides a similar target motion model in each fusion node $w \in\{1, \ldots, W\}$. It is given by:

$$
\mathbf{x}_{k}=\mathbf{F}\left(\Delta_{k}\right) \mathbf{x}_{k-1}+\mathbf{b}\left(\Delta_{k}\right)
$$

where

$$
\mathbf{F}\left(\Delta_{k}\right)=\sum_{w=1}^{W} \nu_{k}^{w} \mathbf{F}_{w}\left(\Delta_{k}\right)
$$

and

$$
\mathbf{b}\left(\Delta_{k}\right)=\sum_{w=1}^{W} \nu_{k}^{w} \mathbf{b}_{w}\left(\Delta_{k}\right)
$$

The equivalent error covariance is given by:

$$
\mathbf{Q}\left(\Delta_{k}\right)=\sum_{w=1}^{W} \nu_{k}^{w 2} \mathbf{Q}_{w}\left(\Delta_{k}\right)
$$

This model is used to predict each track $\mathcal{T}_{k, l}^{w}$ and realise the track-to-track association process.

4. Each global track $\mathcal{T}_{k, l}^{w}$ for the set of the $W$ fusion nodes are predicted and associated to local estimated state. We use Mahalanobis' distance to calculate the association cost, and to obtain the optimized track association scenario by using a GNN (Global Near Neighbour) ${ }^{8}$ approach. The GNN algorithm is based on the Munkres algorithm.

5. Global level fusion in each fusion node, the fused estimated tracks are obtained in each fusion node by the herein formulas :

$$
\hat{\mathbf{x}}_{k \mid k}=\mathbf{P}_{k \mid k}\left(\mathbf{P}_{k \mid k-1}^{-1} \hat{\mathbf{x}}_{k \mid k-1}+\sum_{w=1}^{W} \Delta \hat{\mathbf{x}}_{k \mid k}^{w}\right)
$$

and

$$
\mathbf{P}_{k \mid k}^{-1}=\mathbf{P}_{k \mid k-1}^{-1}+\sum_{w=1}^{W} \Delta \mathbf{P}_{k \mid k}^{w}
$$

where $\hat{\mathbf{x}}_{k \mid k-1}$ and $\mathbf{P}_{k \mid k-1}^{-1}$ are derived from the global model node 22$\}$ and $\Delta \hat{\mathbf{x}}_{k \mid k}^{w}, \Delta \mathbf{P}_{k \mid k}^{w}$ are the incremental information emitted on the network by each fusion node $w$ in the manner that $(\forall w \in\{1, \ldots, W\}$ :

$$
\Delta \hat{\mathbf{x}}_{k \mid k}^{w}=\mathbf{P}_{k \mid k}^{w}{ }^{-1} \hat{\mathbf{x}}_{k \mid k}^{w}-\mathbf{P}_{k \mid k-1}^{w}{ }^{-1} \hat{\mathbf{x}}_{k \mid k-1}^{w}
$$

and

$$
\Delta \mathbf{P}_{k \mid k}^{w}=\mathbf{P}_{k \mid k}^{w}{ }^{-1}-\mathbf{P}_{k \mid k-1}^{w}{ }^{-1}
$$

Finally, in each fusion node $w$ we obtain the same tracks represented by the global states 26 . Those global states are constrained on the road network.

\subsection{Track fusion in hierarchical architecture}

The last studied architecture is hierarchical. Each fusion node $w$ produces tracks $\mathcal{T}_{k, l}^{w}$ at current time. A master fusion node receives the tracks in order to build global tracks and establish the situation assessment to present the picture to the $\mathrm{C} 2$. The data fusion process follows three steps :

1. Prediction step in the master fusion node. At a current time $k$, each global states $\mathbf{x}_{k-1 \mid k-1}^{G}$ and its associated covariance $\mathbf{P}_{k-1 \mid k-1}^{G}$ are predicted according a classical motion model. We use here the constrained motion model (5). 


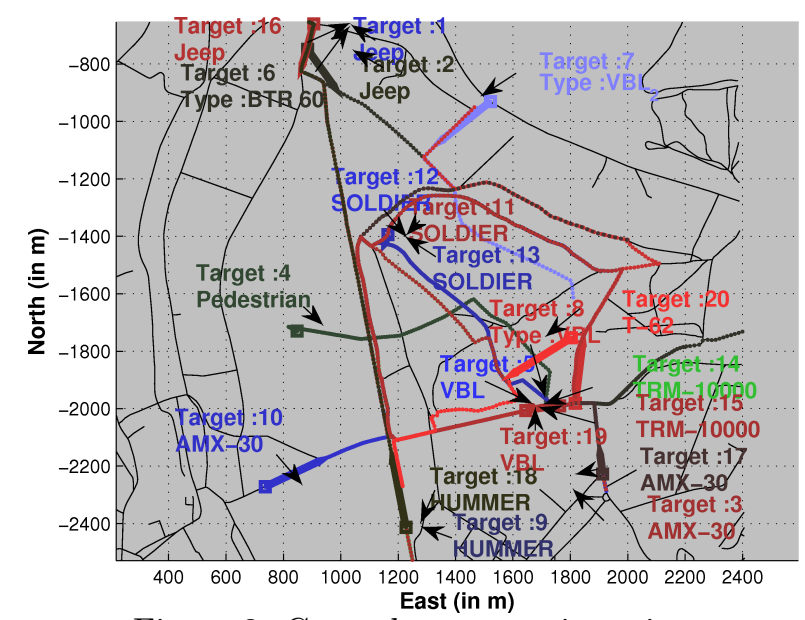

Figure 3: Ground target trajectories.

2. Reception local track $\mathcal{T}_{k, l}^{w}$ step. At time $k$, we calculate the innovation $\mathbf{I n}_{k}^{G}$ and its covariance $\mathbf{S}_{k}^{G}$ to obtain the Mahalanobis distance.

$$
\mathbf{I n}_{k}^{G}=\mathbf{x}_{k \mid k}^{w}-\mathbf{x}_{k-1 \mid k}^{G}
$$

and

$$
\mathbf{S}_{k}^{G}=\omega^{-1} \mathbf{P}_{k \mid k-1}^{G}+(1-\omega)^{-1} \mathbf{P}_{k \mid k}^{w}
$$

where $\omega$ is the fusion factor obtained according with the Information Fast Trace $\frac{14}{14}$ As the distributed architecture, all feasible association between global tracks and local tracks are computed to obtain the cost functions. The similar GNN algorithm is used to obtain the optimized track-to-track association scenario.

3. Global state updated step. The previous step gives the better association between global tracks end local tracks. The global state is updated with the following expression :

$$
\mathbf{x}_{k \mid k}^{G}=\mathbf{x}_{k-1 \mid k}^{G}-W_{k} \mathbf{x}_{k \mid k}^{w}
$$

and

$$
\mathbf{P}_{k \mid k}^{G}=\omega^{-1} \mathbf{P}_{k-1 \mid k}^{G}-W_{k} \mathbf{S}_{k}^{G} W_{k}^{-1}
$$

where the gain $W_{k}$ is defined by:

$$
W_{k}=\omega^{-1} \mathbf{P}_{k-1 \mid k}^{G} \mathbf{S}_{k}^{G-1}
$$

If the altitude component of $\mathbf{x}_{k \mid k}^{G}$ is inferior than 2 meters we consider that the global track is on the ground. Each ground global track in the master node is projected on the road network, to constrain the tracking.

\section{SIMULATION AND RESULTS}

In order to evaluate the three architecture applied to our WSN, we have simulated a realistic complex scenario. In our scenario, we have considered 20 ground targets moving on a chosen operational area. The targets are maneuvering on and off the road network. We distinguish several target types (as tank, jeep, soldiers, civilian pedestrians, etc). Our simulator constrained the maneuvers by taking into account the target type. We have had soldiers (targets number 11, 12,13), and ground vehicles (targets number 14, 15, 19) that move on the battlefield in a close formation. The figure 3 shows the targets trajectories on the area of interest. In this scenario, we also consider terrain masks, due to buildings, vegetation and terrain elevation.

Sensors and fusion nodes are placed at strategic location and to respect with communication constraint: $f^{7}$ (at some road intersections, or in order to get a maximum detection area) to ensure infrastructure protection

\footnotetext{
${ }^{\ddagger}$ We are not allowed to give more details about sensors characteristics in this paper.
} 


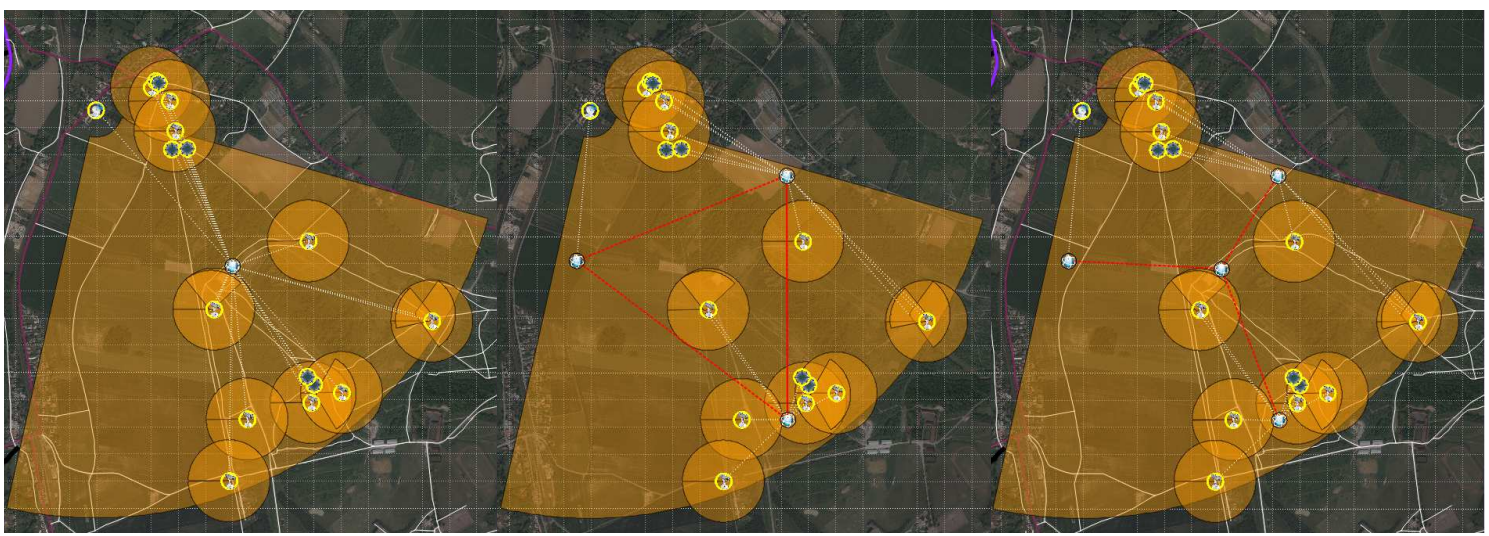

(a) Centralised architecture.

(b) Ditributed architecture.

(c) Hierarchical architecture.

Figure 4: Tested data fusion architectures.

mission. Radar, video and acoustic sensors generate false alarms. The three architectures : centralized , distributed and hierarchical; are tested with three fusion node configurations, represented respectively in figures $4 \mathrm{a}$, $4 \mathrm{~b}$ and $4 \mathrm{c}$. To compute the performance metrics, an important step is to decide at each time which track to compare with which target. In addition, this decision is made in the presence of closely spaced targets and false measurements. The assignment is required to be unique, i.e. at most one track can be associated with one target at any time, and at most one target can be associated with one track. To solve this assignment, Munkres algorithm has been used. The tracks not associated or correlated to a target despite the assignment are considered as false tracks.

The MOP that have been used in this study are the following:

- Root Square Error (RSE). The root square error is the most well-known MOP. It provides an information on the track precision in location and velocity.

- Track Length Ratio (TLR). The track length ratio is a ratio between the track length associated to a target with the length of the target trajectory. It informs on the track continuity performances

The results obtained on the simulated scenario are given in the table 2 ,

Globally, we observe confirmed results on the centralized architecture performances : the centralized process provides better results in precision and track continuity than distributed and hierarchical architectures. In fact, the "optimal" approach (the centralized architecture) uses our VS IMMC SB-MHT to track the targets. Each fusion node uses this algorithm to update track with sensor data, but the fusion process between tracks is done without context information. So, we loose in track precision and track continuity. This phenomenon is accentuated by track-to-track association at road intersection, because we delayed the decision with a sequential test in the SB-MHT, but we take a decision at each time with the GNN algorithm in the distributed and hierarchical architectures.

In addition, the distributed fusion performances are significantly different to hierarchical fusion. This due to the track fusion approach. In fact, the fusion node who receive sensor data are similar. The master node fuses tracks with a global motion model in the hierarchical architecture, whereas in the distributed fusion we keep the constraint motion model information to fuse tracks. The fused estimator seems to be more precise, that brings about a better data association in this multiple target context.

More investigations allow to note a track initialisation problem in the WSN. The track initialisation is done in each fusion node with the VS IMMC SB-MHT. The only confirmed tracks are emitted in order to be fused in upper fusion level. However, we work with heterogeneous sensors and some sensors provide bearing-only measurement (see part 2. If a target moves in a bearing-only sensor wide area and if no track is associated to the target, the fusion node associated to this sensor is unable to initialised a track with the only bearing 


\begin{tabular}{|c||c|c||c|c||c|c|}
\hline \multicolumn{1}{|c||}{} & \multicolumn{2}{c||}{ Centralized } & \multicolumn{2}{c||}{ Distributed } & \multicolumn{2}{c|}{ Hierarchical } \\
\hline Target number & RSE (in m) & TLR & RSE (in m) & TLR & RSE (in m) & TLR \\
\hline 1 & 10.48 & 0.99 & 7.97 & 0.55 & 10.49 & 0.30 \\
\hline 2 & 7.43 & 1 & 7.42 & 1 & 8.58 & 0.87 \\
\hline 3 & 5.32 & 1 & 5.96 & 0.96 & 7.45 & $0 ; 55$ \\
\hline 4 & 6.20 & 0.88 & 6.37 & 0.62 & 6.14 & 0.57 \\
\hline 5 & 6.35 & 1 & 5.08 & 0.98 & 6.74 & 0.33 \\
\hline 6 & 5.66 & 0.68 & 5.41 & 0.51 & 5.48 & 0.55 \\
\hline 7 & 22.45 & 0.6 & 23.54 & 0.65 & 22.97 & 0.66 \\
\hline 8 & 3.43 & 1 & 5.65 & 0.68 & 5.93 & 0.65 \\
\hline 9 & 7.07 & 0.86 & 6.38 & 0.58 & 5.96 & 0.70 \\
\hline 10 & 12.79 & 0.63 & 10.17 & 0.58 & 11.22 & 0.79 \\
\hline 11 & 6.72 & 0.82 & 4.68 & 0.92 & 3.27 & 0.74 \\
\hline 12 & 6.58 & 0.81 & 5.035 & 0.93 & 3.92 & 0.74 \\
\hline 13 & 8.56 & 0.51 & 7.57 & 0.61 & 9.81 & 0.52 \\
\hline 14 & 1.91 & 1 & 1.66 & 1 & 8.65 & 0.78 \\
\hline 15 & 10.39 & 0.37 & 7.26 & 0.49 & 7.23 & 0.12 \\
\hline 16 & 12.77 & 0.34 & 7.93 & 0.33 & 8.08 & 0.08 \\
\hline 17 & 6.44 & 0.78 & 9.21 & 0.87 & 9.79 & 0.86 \\
\hline 18 & 9.30 & 0.48 & 7.39 & 0.52 & 8.46 & 0.28 \\
\hline 19 & 7.06 & 0.6 & 7.72 & 0.34 & 8.63 & 0.35 \\
\hline 20 & 18.55 & 0.38 & 9.75 & 0.46 & 9.71 & 0.23 \\
\hline
\end{tabular}

Table 2: Synthetic MOP.

measurement. So, if a global track is in this configuration, the global track can't be fused and updated because no local track can't be emitted by the fusion node.

Despite of the group class information given by video and acoustic sensors, the different architectures can't achieve to track soldier's group. This is due to the heterogeneous measurement model. A group is only one detection for the previous sensors that brings about a track initialisation. But with radar sensor a group can be several detections due to resolution cell. An ambiguity arises in track association if several heterogeneous sensor detect a group resulting track lost. The different studies of network architectures can't solve this problem. A solution must be found at the local fusion node and shared for the track fusion.

For each architectures, the results obtained on integrated solution (hardware environment) show the on-boarded constraints (not detailed in this paper) are satisfied.

\section{CONCLUSION}

To conclude this work, we have proposed three multiple target tracking schemes in a Wireless Sensor Network (WSN). Those schemes implement centralized, distributed and hierarchical architectures for a fusion node configuration. Each fusion node is associated to a set of sensor and the measurement are treated by our VS IMMC SB-MHT. This multiple target tracking algorithm is able to track several moving targets by taking into account the road network information and the terrain elevation to improve the track continuity. However, because of communication constraint a centralized architecture can't be an operational solution. So we have studied alternative solutions by developing distributed and hierarchical algorithms for our WSN. The results show differences between the performances. This is a preliminary work and more investigations must be done to improve each fusion architecture to select the best one for the mission goal. However, if the on-boarded conditions are respected, several points must be studied before the system validation : $\mathrm{n}$ details before validating the system for operational needs, in particular complementary studies must be carried out to detect and track groups of targets through the WSN, to initialize tracks at each local fusion node with a global track, correlate the survivals tracks with old tracks to improve the track continuity, study the automatic sensor calibration in WSN with multiple target and validate the results in operational condition. 


\section{REFERENCES}

1. Ekman, M. and Palsson, H., "Ground target tracking of vehicles in a wireless sensor network," Proc. of FUSION 2012, Conf., Singapore (2012).

2. Ekman, M. and Sviestins, E., "Mutiple model algorithm based on particle filters for ground target tracking," in Proc. of FUSION 2007, Conf., Quebec, July 2007. (2007).

3. D. Salmond, M. Clark, R. V. and Godsill, S., "Ground target modelling, tracking and prediction with road networks," in Proc. of FUSION 2007, Conf., Quebec, July 200\%. (2007).

4. Parmar, P. and Zaveri, M., "Mutiple target tracking and data association in a wireless sensor network," in Proc. of fourth international conference on computational intelligence and communications network 2012 (2012).

5. S. Oh, S. S. and Shenato, L., "A hierarchical multiple-target tracking algorithm for sensor networks," in Proc. of the 2005 IEEE international conference on robotics and automation, Conf., Barcelona, Spain (2005).

6. Ulmke, M. and Koch, W., "Road-map assisted ground moving target tracking," IEEE transactions on Aerospace and Electronic Systems 42, 1264-1274 (2006).

7. Pannetier, B. and Dezert, J., "Track segment association with classification information," Sensor Data Fusion: Trends Solutions Applications (SDF), Workshop on.

8. Blackman, S. and Popoli, R., [Design and analysis of modern tracking systems], Artech House (1999).

9. B. Pannetier, J. D. and Pollard, E., "Improvement of multiple ground targets tracking with gmti sensor and fusion of identification attributes," IEEE aerospace conference.

10. yang, C. and Blasch, E., "Kalman filtering with nonlinear state constraints," IEEE transactions on Aerospace Electronic Systems 45, 70-84.

11. Y. Bar-Shalom, X.-R. L. and Kirubarajan, T., [Estimation with Applications to Tracking and Navigation], John Wiley and Sons, New York (2001).

12. B. Pannetier, V. N. and Rombaut, M., "Multiple ground target tracking with a gmti sensor," Proceedings of MFI.

13. Ding, Z. and Hong, L., "A distributed imm fusion algorithm for multi-platform tracking," Signal Processing 64, 167-176.

14. Franken, D. and Hupper, A., "Improved fast covariance intersection for distributed data fusion," in Proc. of FUSION 2005, Conf., Philadelphia, July 2005. (2005). 